Research Paper

\title{
Dihydromyricetin Inhibits Tumor Growth and Epithelial-Mesenchymal Transition through regulating miR-455-3p in Cholangiocarcinoma
}

\author{
Xin Li1 ${ }^{1,2}$, Zhou-Sheng Yang 3 , Wen-Wu Cai ${ }^{4}$, Yang Deng, ${ }^{5}$, Lei Chen ${ }^{6,7 凶}$ and Sheng-Lan Tan ${ }^{6,7 凶}$ \\ 1. Department of Vascular Surgery, the Second Xiangya Hospital, Central South University, Changsha, Hunan, China, 410011. \\ 2. The Institute of Vascular Diseases, Central South University, Changsha, Hunan, China, 410011. \\ 3. Department of Pharmacy, The People's Hospital of Guangxi Zhuang Autonomous Region, Nanning, Guangxi, China, 530021. \\ 4. Department of General Surgery, The Second Xiangya Hospital, Central South University, Changsha, China, 410011. \\ 5. Department of pharmacy, The Third Hospital of Changsha, Changsha, China, 410015. \\ 6. Department of Pharmacy, The Second Xiangya Hospital, Central South University, Changsha, China, 410011. \\ 7. Institute of Clinical Pharmacy, Central South University, Changsha, China, 410011.
}

$\triangle$ Corresponding authors: Lei Chen, E-mail: mikelei@csu.edu.cn, Phone Number: +86-0731-8529-2072, Fax Number: +86-0731-8529-2074 and Sheng-Lan Tan, E-mail: sltan@csu.edu.cn, Phone Number: +86-0731-8529-2072, Fax Number: +86-0731-8529-2074.

(C) The author(s). This is an open access article distributed under the terms of the Creative Commons Attribution License (https://creativecommons.org/licenses/by/4.0/). See http://ivyspring.com/terms for full terms and conditions.

Received: 2021.04.06; Accepted: 2021.08.10; Published: 2021.08.22

\begin{abstract}
Cholangiocarcinoma (CCA) leads to poor prognosis due to high aggressiveness and common chemoresistance. Dihydromyricetin (DMY), the main bioactive compound isolated from Ampelopsis grossedentata, exhibits broad anti-tumor effects. This study aimed to investigate the inhibitory effect of DMY on CCA tumor growth and epithelial-mesenchymal transition (EMT) and its underlying mechanism in CCA. DMY treatment significantly inhibited cell proliferation and EMT in CCA cell lines. The expression of ZEBI and vimentin were down-regulated, while the level of E-cadherin was increased after DMY treatment. By analyzing the TCGA dataset, we found that miR-455 expression was significantly downregulated, while the level of ZEBI was up-regulated in human CCA tumor tissues compared to normal samples. Mechanistic studies showed that ZEBI was a direct target of miR-455-3p in CCA. Moreover, DMY treatment potently increased miR-455-3p expression and inhibited ZEBI expression. Inhibition of miR-455-3p expression abolished DMY's inhibitory effects on tumor growth and EMT in both CCA cells and cell-engrafted nude mice. Finally, DMY significantly suppressed the expressions of $\mathrm{p}-\mathrm{PI} 3 \mathrm{~K}$ and $\mathrm{p}-\mathrm{AKT}$, while silencing miR-455-3p remarkably abrogated the inhibitory effect. In conclusion, DMY suppresses tumor growth and EMT through regulating miR-455-3p in human cholangiocarcinoma, suggesting a potential option for CCA treatment.
\end{abstract}

Key words: Dihydromyricetin, cholangiocarcinoma, miR-455-3p, epithelial-mesenchymal transition

\section{Introduction}

Constituting approximately $15 \%$ of all primary liver tumors, cholangiocarcinoma (CCA) is the second most common primary hepatic malignancy after carcinoma [1]. According to their anatomical locations, CCAs are divided into three categories as intrahepatic (iCCA), perihilar (pCCA), and distal (dCCA). The incidence and mortality of CCA are increasing globally in the past decades, placing a worldwide health problem [2]. The prognosis of CCA patients is still disappointing, with low 5-year survival $(7-20 \%)$ [2]. Surgery is the best option for CCA, however, up to $70 \%$ patients are diagnosed at late stages due to no specific symptoms, resulting in no chance of surgery and can only receive palliative treatment $[2,3]$. The cisplatin plus gemcitabine chemotherapy is the first-line regimen for patients with advanced CCA. Besides a lot of side effects, the major limitation of chemotherapy in the management of patients with advanced CCA is the marked multi-drug resistance phenotype of CCA [2]. Therefore, studies of new therapeutic agents for CCA treatment are urgently required to improve patient medical outcomes.

Due to the unsatisfactory effect of traditional cytotoxic drugs in the treatment of CCA, it is promising to develop new drugs based on other 
mechanisms of tumor pathogenesis. Epithelial to mesenchymal transition (EMT) is defined as a cell biological program, during which epithelial cells progressively lose their junctions and apical-basal polarity, and gradually obtain mesenchymal characteristics [4]. EMT is a relatively reversible process and mesenchymal-epithelial transition (MET) describes the reverse procedure. Accumulating evidences demonstrate that EMT not only plays an important role in embryogenesis, organ development, tissue regeneration and organ fibrosis, but also contributes greatly to cancer development and progression [5, 6]. For instance, cancer cells promote their ability of migration, invasion and metastasis via the mechanism of EMT. Moreover, EMT is also involved in reducing sensitivity of cancer cells to chemotherapy. During EMT, cancer cells alter a series of gene expressions, including downregulation of epithelial markers (e.g. E-cadherin) and upregulation of mesenchymal markers (e.g. N-cadherin and vimentin) [4].

Natural plant compounds have been used for decades as adjuvant therapy and a structural basis for drug development. Many bioactive compounds isolated from natural plants can regulate EMT through the mechanisms of anti-inflammation, anti-fibrosis or antioxidation [7]. Dihydromyricetin (DMY), a natural flavonoid and the main active compound isolated from the plant Ampelopsis grossedentata, has broad spectrum and multiple target effects in several cancers with low toxicity, suggesting a high and promising possibility of DMY in cancer treatment [8-10]. We previously found DMY significantly inhibited CCA cell migration and invasion via inhibiting miR-21 expression [11]. Yet, overexpression of miR-21 only partly abrogated the DMY's anti-tumor effects in CCA, which promotes us to discover a comprehensive mechanism of how DMY exerts its anti-tumor effects.

MicroRNAs (miRNAs) are single stranded non-coding RNAs with a length of 19 to 24 nucleotides that negatively regulate the expression of target genes. Studies have demonstrated that multiple miRNAs are involved in cancer metastasis and progression through controlling EMT processes including in CCA [12]. For instance, a previous study showed that miR-186 inhibited CCA cell proliferation and EMT through targeting Twist1 [13]. MiR-455 has been shown to be down-regulated in several gastrointestinal cancers, including gastric cancer and pancreatic cancer [14, 15]. However, the role of miR-455 in CCA is still largely unknown. By analyzing the TCGA dataset, we identified that the expression of miR-455 in human CCA tissues was also significantly decreased compared to the non-tumor tissues. As the previous study showed that miR-455 exerted anti-cancer effects in non-small cell lung cancer through targeting the EMT inducible transcription factor ZEB1 [16], we aimed to investigate if the miR-455/ZEB1 axis was also involved in EMT in CCA in this study.

In the current study, we investigated the effects of DMY on tumor growth and EMT in human CCA both in vitro and in vivo. For the first time, we found that DMY significantly inhibited tumor growth and EMT by targeting the miR-455/ZEB1 axis, suggesting DMY might be explored as a potential candidate for the treatment of CCA.

\section{Material and Methods}

\section{Cell culture and treatment}

Human CCA cell lines including RBE and TFK-1 were purchased from American Type Culture Collection (Rockville, MD, USA). The cells were maintained in RPMI-1640 medium (Gibco, Thermo Fisher Scientific, MA, USA) containing 10\% fetal bovine serum (Gibco) in a humidified atmosphere at $37{ }^{\circ} \mathrm{C}$ with $5 \% \mathrm{CO}_{2}$. Different concentrations of dihydromyricetin (Sigma-Aldrich, St Louis, MO, USA) dissolved in dimethyl sulfoxide (DMSO, Sigma-Aldrich) were tested and $150 \mu \mathrm{M}$ was finally selected for treatment.

\section{Cell viability assay}

The Cell Counting Kit-8 (CCK-8) (Dojindo, Japan) assay was used to assess cell viability after exposure to different concentrations of dihydromyricetin. Briefly, RBE cells were seeded in 96-well plates at a density of $5 \times 10^{4}$ cells per well and were treated with different concentrations of dihydromyricetin $(10,50,100,150,200$ and $400 \mu \mathrm{M})$ for 24 hours. After treatment, cell culture medium was replaced with fresh medium containing $10 \mu \mathrm{L}$ CCK-8 solution in each well, and cells were incubated for $1 \mathrm{~h}$ at $37^{\circ} \mathrm{C}$ with $5 \% \mathrm{CO} 2$. The absorbance of the solution was measured at $450 \mathrm{~nm}$ using a Microplate Reader (Bio-Rad, CA, USA).

\section{Colony formation assay}

Suspensions of single RBE cells were prepared and seeded in six-well plates. Cells were maintained in RPMI-1640 supplemented with $10 \%$ fetal bovine serum containing different treatment solution at $37^{\circ} \mathrm{C}$ and 5\% CO2 for about 2 weeks. When single-cell clones become visible, the colonies were washed with PBS twice, followed by fixed in $4 \%$ paraformaldehyde for $15 \mathrm{~min}$. Then the colonies were stained with crystal violet (Beyotime, Beijing, China). Finally, colonies were counted under a microscope (Olympus Corp., Tokyo, Japan). 


\section{Cell proliferation assay}

The EdU assay (Beyotime, Beijing, China) was used to measure cell proliferation according to the manufacturer's instructions. Cells were plated in 96-well plates at a density of $5 \times 10^{4}$ cells per well. After treatments, cell culture medium was replaced with pre-warmed fresh medium containing $10 \mu \mathrm{M}$ EdU, and cells were incubated for $3 \mathrm{hrs}$. Then cells were fixed in $4 \%$ Paraformaldehyde for $15 \mathrm{~min}$ and incubated in $0.3 \%$ Triton- $X 100$ for $15 \mathrm{~min}$ at room temperature. After washed with PBS for three times, cells were incubated with the prepared Click buffer for $30 \mathrm{~min}$ in dark at $37{ }^{\circ} \mathrm{C}$, followed by counterstained with Hoechst for $10 \mathrm{~min}$. Finally, EdU-positive cells, DAPI-labeled cells and their merged images were captured under a fluorescence microscope (Zeiss, Jena, Germany).

\section{Cell invasion and migration assays}

Transwell chambers with $8 \mu \mathrm{m}$ size of pores (BD Biosciences, CA, USA) were used to assess the migration and invasion of cells same as our previous study [11]. For cell invasion evaluation, chambers were coated on the upper side with BD MatrigelTM Matrix (BD). After treatment, $8 \times 10^{4}$ cells suspended in $200 \mu \mathrm{l}$ of serum free medium were added to the upper chamber, and the lower chambers were supplemented with medium containing 10\% FBS. After incubated for $24 \mathrm{hrs}$, cells remaining in the upper chamber were completely removed by gently swabbing. Cells which migrated to the lower chamber were fixed with $4 \%$ paraformaldehyde for $15 \mathrm{~min}$ and stained with $0.4 \%$ crystal violet (Merck, Germany) for 15 min. Finally, cells were counted and photographed under microscope. The method of cell migration was similar, except using chambers without BD Matrigel.

\section{RNA isolation and quantitative real-time polymerase chain reaction}

RNA level was measured by RT-qPCR. Total RNA was extracted from tumor tissues and cells by TRIzol regent (Invitrogen, CA, USA) and then reversely transcribed into cDNA. The commercial Reverse Transcribed Kit (Takara, Dalian, China) was used to synthesize cDNA, and real-time qPCR was conducted using the TB Green real-time PCR kit (Takara, Dalian, China). U6 was used as an internal control. The primer sequences used were: ZEB1 forward, 5'-GCACCTGAAGAGGACCAGAG-3'; ZEB1 reverse, 5'-GTGTAACTGCACAGGGAGCA-3'. glyceraldehyde-3-phosphate dehydrogenase (GAPDH) forward, 5'-CTGCACCACCAACTGCT TAG-3', reverse, 5'-AGGTCCACCACTGACAC GTT-3'. The primers for miR-455-3p (MQPS00014641-100) and U6 (MQPS0000002-1-100) were ordered from RiboBio (Guangzhou, China).

\section{Screening of differentially expressed genes in human CCA}

The RNA-seq and miRNA-seq data of cholangiocarcinoma were downloaded from the TCGA database. The $\mathrm{R}$ software was used to standardize and $\log 2$ transform the data. TCGA ID was used to match the expression of ZEB1 and miR-455. Spearman correlation coefficient was calculated and statistically tested. The results were displayed with R package "ggpubr".

\section{Cell transfection}

RBE cells were transfected with miRNA nonspecific mimic control (NS-m) (miR1N0000001-1-5, Ribobio, Guangzhou, China), miR-455-3p mimic (455-m) (miR10004784-1-5, Ribobio, Guangzhou, China), miRNA non-specific inhibitor (NS-i) (miR2N0000001-1-5, Ribobio, Guangzhou, China), miR-455-3p inhibitor (455-i) (miR20004784-1-5, Ribobio, Guangzhou, China) at a final concentration of $100 \mathrm{nM}$ following the manufacturer's protocol. Cells were transiently transfected by use of Lipofectamine 2000 transfection reagent from Invitrogen (11668019, USA). After 14 to16 hrs of incubation, fresh culture medium was replaced and cells were incubated for another 12 hours before harvested for protein or RNA analyses. Real time qPCR was used to confirm that miR-455 expression was specifically up-regulated or down-regulated after transfection.

\section{Western blot assay}

Tumor tissues were first homogenized using TissueLyser II (Qiagen, USA) according to manufacturer's instructions. Then proteins from homogenized tumor tissues and CCA cell lines were lysed with the radio immunoprecipitation assay (Beyotime, Beijing, China). Proteins were separated by sodium dodecyl sulfate-polyacrylamide gel electrophoresis (SDS-PAGE) using a 10\% gradient gel and transferred onto $0.45 \mu \mathrm{m}$ polyvinylidene fluoride (PVDF) membranes (Millipore, Billerica, MA, USA). After blocking nonspecific binding with TBS-T $(0.1 \%$ tween) containing 5\% non-fat milk for $1 \mathrm{~h}$ at room temperature, the membranes were incubated with primary antibodies at $4{ }^{\circ} \mathrm{C}$ overnight and then secondary antibodies for $1 \mathrm{~h}$ at room temperature. The protein bands were detected using electrochemiluminescence (ECL) (Beyotime, Beijing, China), and band quantification was analyzed using the Image $\mathrm{Lab}^{\mathrm{TM}}$ Software (Bio-Rad, USA). The primary antibodies used in this study were as follows: ZEB1 (1:1000, 21544-1-AP, Proteintech, China), E-cadherin (1:5000, 20874-1-AP, Proteintech, China), vimentin 
(1:1000, 3932, CST, USA), phospho-PI3K (1:1000, AP0854, Abclonal, China), phospho-Akt (1:1000, 4060, CST, USA), and GAPDH (1:1000, ab8245, Abcam, $\mathrm{UK})$.

\section{Dual-luciferase reporter assay}

The wild-type (WT) and mutant-type (Mut) 3'-untranslated regions (UTRs) of ZEB1 were synthesized and cloned to pmirGLO Dual-Luciferase miRNA Target expression vector (E1330, Promega, USA) by Vigene Biosciences (Shandong, China) according to the manufacturer's instructions. HEK 239T cells $\left(1.5 \times 10^{5}\right.$ cells per well $)$ were seeded on 12-well plates and co-transfected with $250 \mathrm{ng}$ WT or Mut luciferase reporter constructs, 10 ng Renilla plasmid (E2231, Promega, USA), 100 nM 455-m or NS-m, together with lipofectamine 2000 after cells growing to $70 \%$ confluency. The cell lysates were collected 24 hours after transfection, and the luciferase activities were measured using the Dual-Glo Luciferase Assay System (E2920, Promega, USA). A fluorescence reader (Veritas 9100-002, Turner Biosystems, USA) was used and each reading of luciferase activity was normalized to the Renilla activity.

\section{Nude mice xenograft tumorigenesis studies}

All animal experiments were approved by the Ethics Committee for Laboratory Animals of The Second Xiangya Hospital of Central South University, Hunan, China, and followed the Interdisciplinary Principles and Guidelines for the Use of Animals in Research, Testing, and Education by the New York Academy of Sciences, Ad Hoc Animal Research Committee. RBE cells $\left(5 \times 10^{6}\right)$ were resuspended in $50 \mu \mathrm{L}$ RPMI-1640 medium mixed with $50 \mathrm{uL}$ Matrigel. Then cells were subcutaneously injected into the right flank of male four-week-old Balb/c nude mice (Hunan SJA Laboratory Animal Cooperation, Changsha, Hunan, China). Three days after injection, mice were randomly divided into two groups $(n=6$ mice per group) and intragastric treated with DMY (500 mg/ kg/day) for 4 weeks. Intratumoral injection with lipofectamine 2000 encapsulated 455-i or NS-i (5 $\mathrm{nM} /$ mouse) was according to protocol previously described [17]. Animals were maintained under specific pathogen-free conditions, and animal protocols were reviewed and approved by the Ethics Committee for Laboratory Animals of The Second Xiangya Hospital, Central South University, Hunan, China. All mice were euthanized 4 weeks after injection, and the xenograft tissues were harvested and fixed with $10 \%$ formalin solution for immunohistology analysis.

\section{Immunohistochemistry}

The tissue paraffin sections were heated in an oven at $60{ }^{\circ} \mathrm{C}$ for $2 \mathrm{~h}$, followed by dewaxed with xylene for $20 \mathrm{~min}$, and dehydrated with gradient ethanol solution. Then sections were washed with PBS three times, incubated in 3\% hydrogen peroxide for 10 min, washed with PBS three times again, and put in sodium citrate buffer (10 nM sodium and 0.05\% Tween 20 at $\mathrm{pH} 6.0$ ) at $96{ }^{\circ} \mathrm{C}$ for 30 minutes for antigen repair. After being blocked with calf serum for $20 \mathrm{~min}$, primary antibodies were added and incubated for 60 minutes at room temperature, including Ki67 (1: 50, A2094, Abclonal), ZEB1 (1:50, 21544-1-AP, Proteintech, China), E-cadherin (1:500, 20874-1-AP, Proteintech, China) and vimentin (1:1000, 3932, CST, USA). After rinsed with PBS three times, the secondary antibodies were incubated at $37^{\circ} \mathrm{C}$ for $30 \mathrm{~min}$, washed with PBS three times, and developed with DAB (ZLI-9017, Zsbio, China). Sections were then counterstained with hematoxylin, dehydrated with ethanol, cleared with xylene, and mounted. All images were captured using a Microscope VS120 Whole Slide Scanner (Olympus) and analyzed using the computer-assisted Image-Pro Plus software (Meida Cybernetics, Bethesda, MD). The quantifications of Ki67, and ZEB1, E-cadherin and vimentin staining were performed as positive area percent. All specimens were confirmed by two independent observers.

\section{Statistical analysis}

All data were analyzed using the SPSS 22.0 statistical software (IBM Corp., NY, USA) and GraphPad Prism 5.01 (GraphPad Software, CA, USA) and data were presented as mean \pm standard deviation. Each experiment was performed in triplicates for at least three times. Comparisons between two groups or multiple groups were performed using the independent t-test or one-way analysis of variance (ANOVA). Spearman correlation was used to calculate the coefficient between miR-455 and ZEB1. ${ }^{*} P<0.05,{ }^{* *} P<0.01$ and ${ }^{* * *} P<0.001$ were considered statistically significant.

\section{Results}

\section{DMY inhibits cell growth and EMT in CCA cell lines}

DMY has shown broad anti-tumor effects in multiple cancers without adverse side effects, therefore, the safety and effect of DMY on cell growth of the RBE cell line was first assessed by the CCK- 8 assay. As shown in Figure S1, DMY inhibited the growth of RBE cells in a dose-dependent manner, and its half maximal inhibitory concentration (IC50) was 
146.6 $\mu \mathrm{M}$. Thus, in the following experiments, we used $150 \mu \mathrm{M}$ of DMY to treat the CCA cell lines. Next, the colony-forming assay was performed and the result demonstrated that DMY suppressed about $70 \%$ of cell growth in RBE cells compared to the controls (Figure 1A). The EdU assay showed that DMY significantly inhibited cell proliferation in RBE cells (Figure 1B). Because one of the most important issues in treating patients with CCA is the high metastatic capability of CCA cells, we attempted to investigate DMY's inhibitory effect on migration and the underlying mechanism in CCA in this study. As epithelial-to-mesenchymal transition (EMT) is one of the most essential mechanisms of enhancing cancer cell migration ability in multiple tumors including CCA $[5,6]$, we performed several functional experiments in the subsequent experiments. As shown in Figure 1C, $150 \mu \mathrm{M}$ of DMY treatment significantly inhibited cell migration and invasion in both RBE cells and TFK-1 cells as determined by the transwell assay. Moreover, Western blotting showed that DMY treatment significantly altered the expression of EMT-related marker genes, including reduced vimentin and ZEB1 protein expressions and increased E-cadherin expression compared to the control group in both RBE and TFK-1 cells (Figure 1D). Collectively, these data suggest that DMY inhibits cell growth and EMT in cholangiocarcinoma cells.

\section{The miR-455-3p/ZEB 1 axis is involved with EMT in CCA and may be the target of DMY}

Accumulating data have proven that miRNAs are potential upstream regulators of EMT in cancer cells, including CCA $[12,13]$. Therefore, we used TargetScan algorithm (http://www.targrtscan.org/; last accessed March 20, 2021) and predicted that ZEB1 was a potential target of miR-455-3p. MiR-455 is involved in development of diverse malignancies, and a previous study in human keratinocyte (HaCaT) cells demonstrated that the miR-455/ZEB1 axis played an important role in EMT during arsenite-induced malignant transformation [18]. Thus, we next investigated whether miR-455/ZEB1 axis-mediated EMT was also involved in CCA and DMY's anti-EMT effects. In order to answer these questions, we first explored the expressions of miR-455 and ZEB1 in human CCA tissues compared to the normal samples by analyzing the TCGA dataset. As shown in Figure 2A, miR-455 expression was significantly decreased in
A

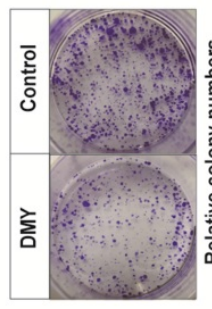

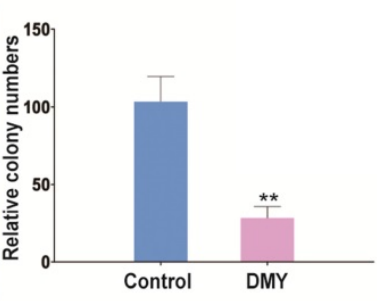

B
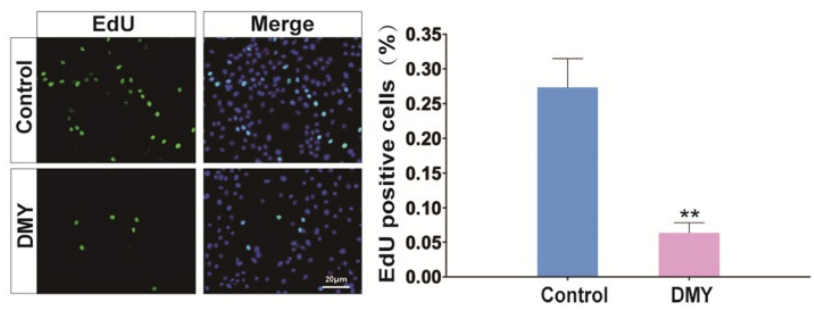

C
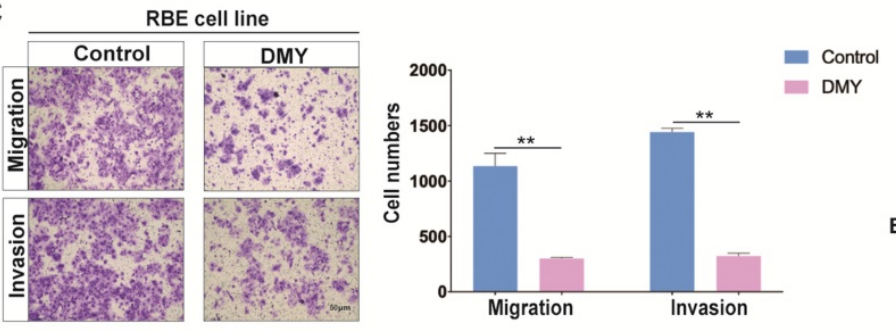

D
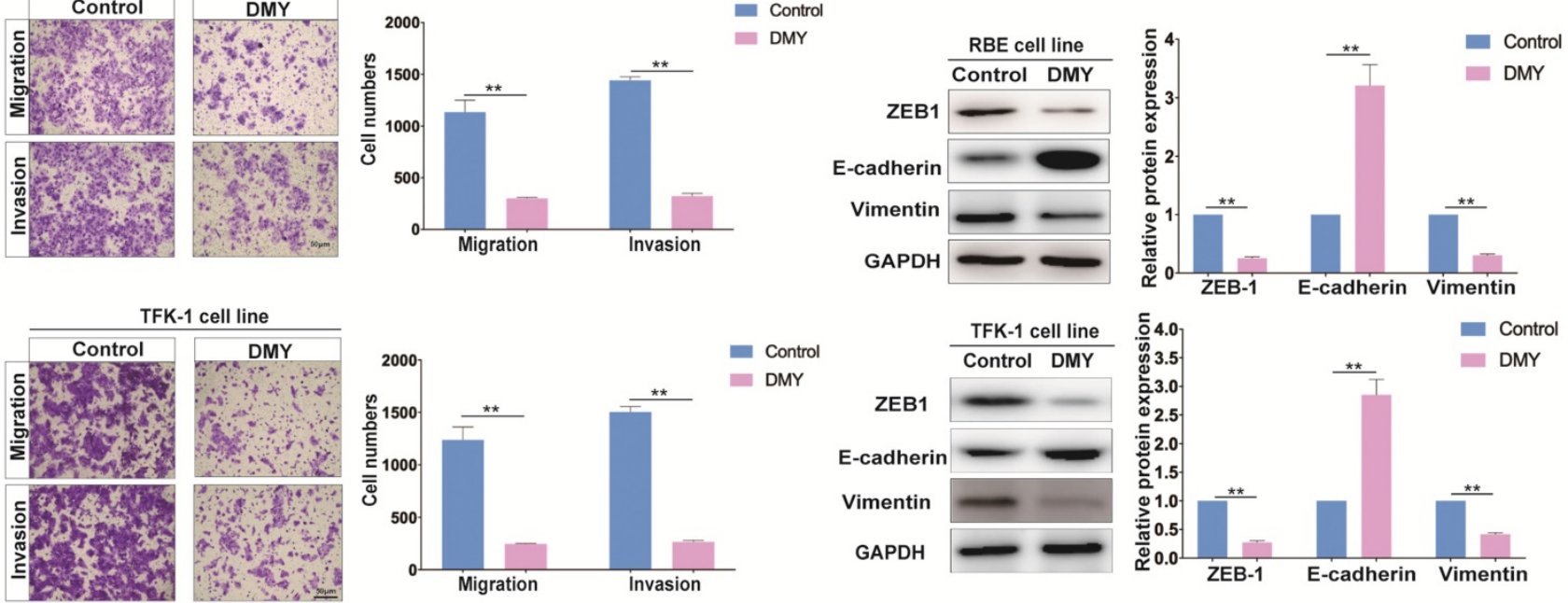

Figure 1. Dihydromyricetin inhibits cell growth and epithelial to mesenchymal transition (EMT) in RBE cells and TFK-1 cells. (A-B) Colony formation assay and EdU assay were used to determine cell growth and proliferation between cells treated with $150 \mathrm{uM}$ of dihydromyricetin and DMSO control solvent in RBE cells. (C) Cell migration and invasion abilities were tested by transwell assay. (D) Western blot assay was performed to determine the levels of proteins (ZEBI, E-cadherin, Vimentin) associated with EMT. $n=3$ independent experiments. Values were given as means \pm SEM. $* * P<0.01$. 
tumor tissue compared to normal tissues in human CCA in a microarray data extracted from TCGA dataset. This observation was further confirmed in a specific data set derived from TCGA webset (Figure 2B). Conversely, the expression of ZEB1 in CCA tissues was remarkably up-regulated (Figure 2C). Moreover, the expression of ZEB1 was negatively related to the expression of miR-455 in CCA tissues (Figure 2D), suggesting that ZEB1 may be a target of miR-455 in CCA.

Next, a luciferase reporter assay was performed to confirm the association between miR-455-3p and ZEB1 in CCA (Figure 3A). As shown in Figure 3B, cells transfected with miR-455-3p mimics (455-m) exhibited a significantly reduced luciferase activity compared to those cells transfected with non-specific mimics (NS-m), indicating that ZEB1 is a direct target of miR-455-3p. Next, we investigated whether miR-455-3p exerted its function through controlling

A

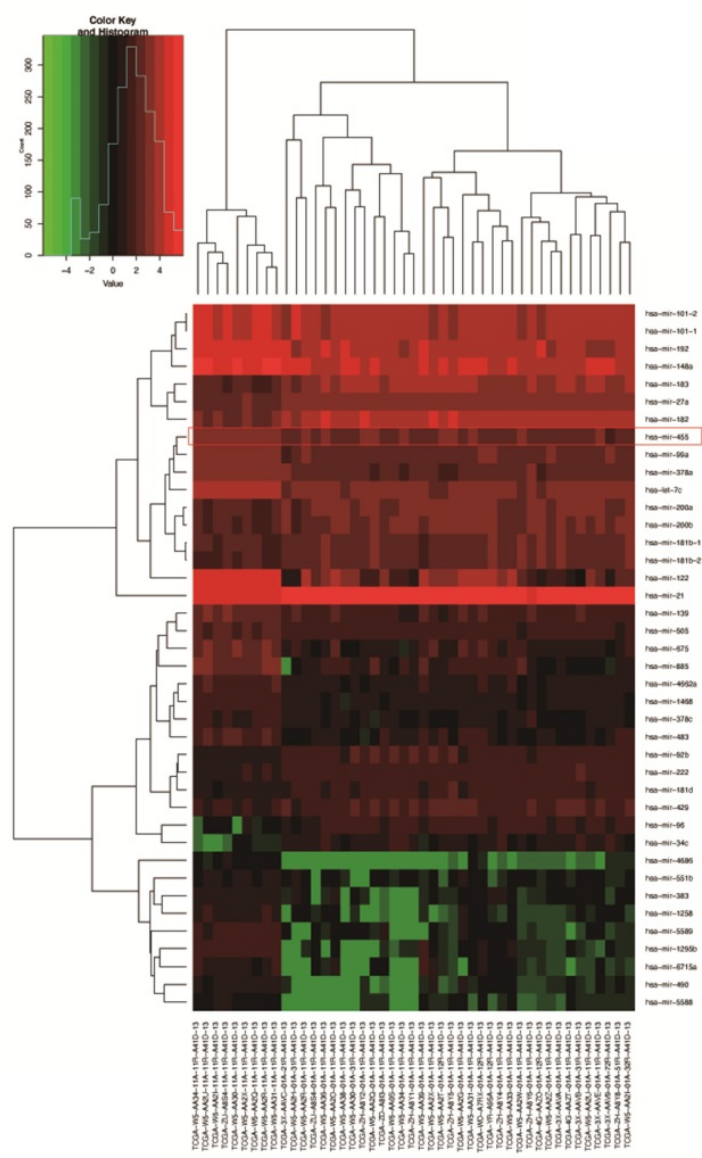

ZEB1 expression in CCA cells. We found that in both RBE and TFK-1 cells which were transfected with 455-m showed significantly reduced ZEB1 expression (Figure 3C). Conversely, down-regulation of miR-455-3p using miR-455-3p inhibitor (455-i) markedly increased the expressions of ZEB1 at both mRNA and protein level (Figure 3D). The efficiencies of 455-m and 455-i were confirmed by qPCR (Figure S2). Moreover, we also tested the expressions of miR-455-3p and mRNA level of ZEB1 after exposure to DMY in both RBE cells and TFK-1 cells as assessed by real time PCR. As shown in Figure 3E, DMY treatment significantly increased the expression of miR-455-3p and decreased the mRNA level of ZEB1 compared to control cells. Taken together, these data demonstrated that ZEB1 is the direct target of miR-455-3p in CCA and the underlying mechanism of DMY mediated-EMT inhibition effects in CCA may be through regulating the miR-455-3p/ZEB1 axis.

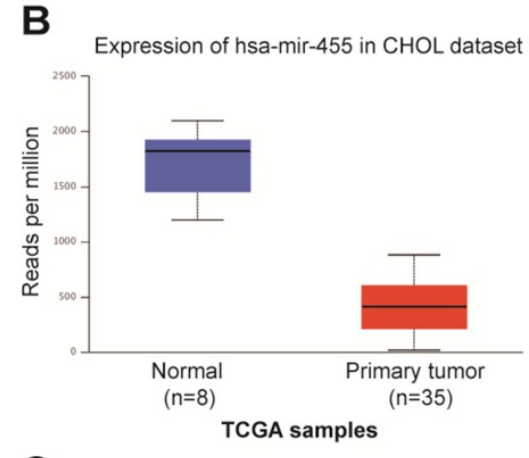

C
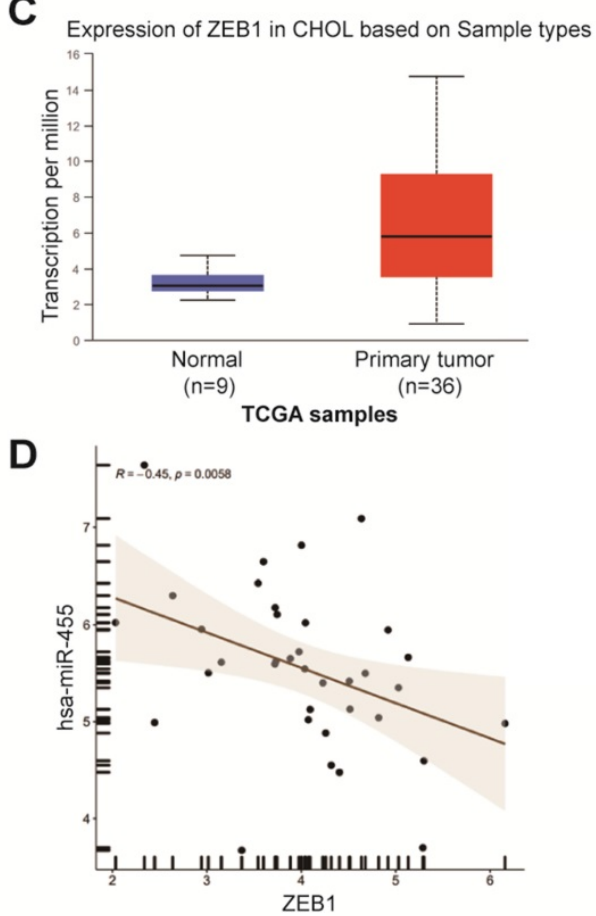

Figure 2. Expression of miR-455 and ZEB1 in human cholangiocarcinoma tissue. (A-C) TCGA dataset for analyzing the expressions of miRNAs, miR-455 and ZEB1 in human cholangiocarcinoma tissue. (D) Expression of ZEB1 was negatively related to the expression of miR-455 as analyzed by the Spearman's correlation analysis. 
A

ZEB1 3' UTR (WT)

hsa-miR-455

ZEB1 3' UTR (Mut) 5' ...CAUUUAUACUUGCCUCUACAGAU... 3'

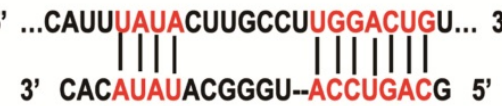

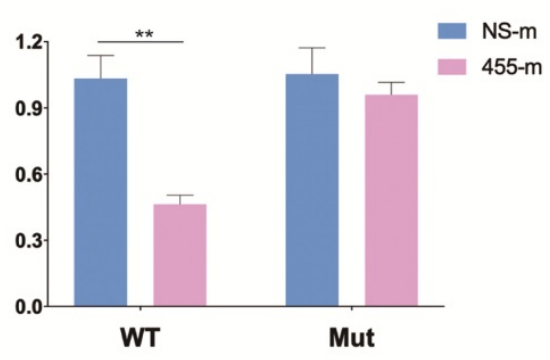

D

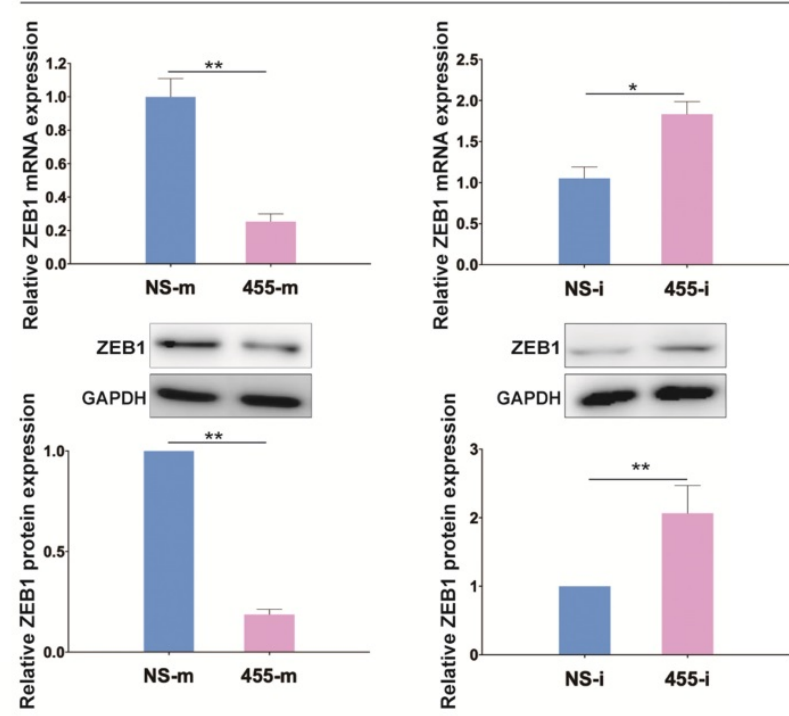

C

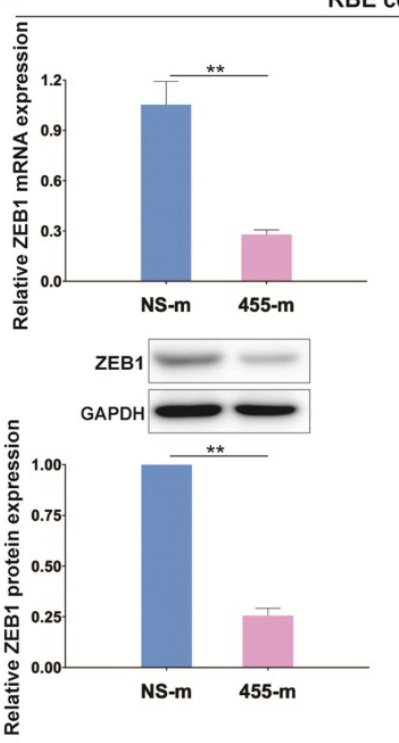

RBE cell line

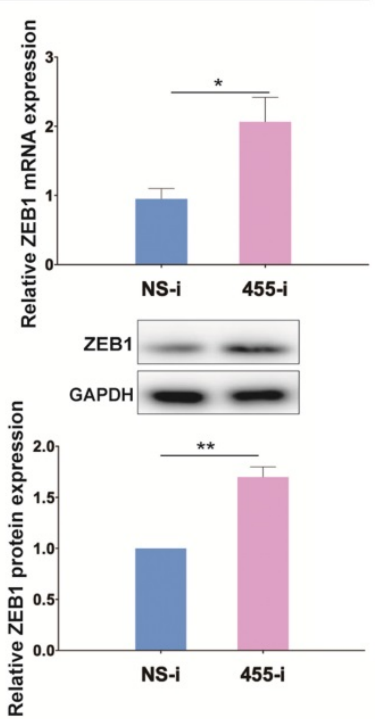

E

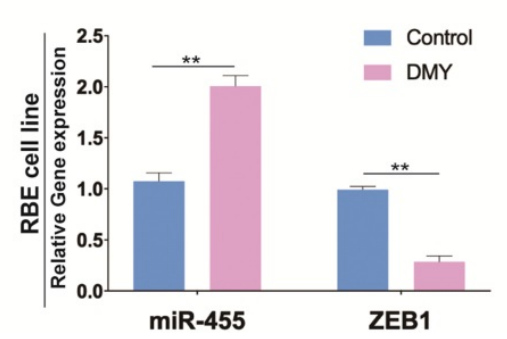

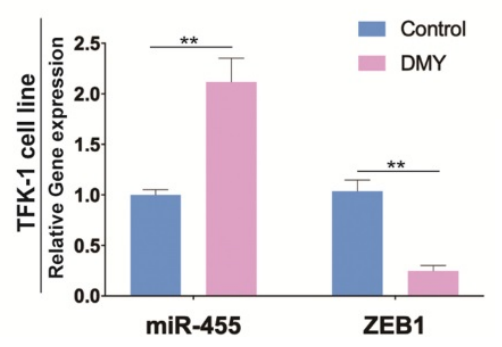

Figure 3. ZEB1 is the target of miR-455 in human cholangiocarcinoma and dihydromyricetin regulates the expressions of miR-455 and ZEB1. (A) Schematic graph illustrating the binding sites of miR-455-3p in the 3'-UTR region of ZEB1. (B) The relative luciferase activity of wild type or mutant type ZEB1 vector was evaluated by Dual-Luciferase reporter assay system in RBE cells. (C-D) In RBE cells and TFK-1 cells, the mRNA level and protein level of ZEB1 in cells transfected with NS-i or 455-i (NS-m or 455-m) were respectively measured by real time PCR and western blotting. (E) Real time PCR was adopted to measure the RNA level of miR-455 and ZEB1 after exposure to 150 uM of dihydromyricetin or DMSO control solvent in RBE cells and TFK-1 cells. $\mathrm{n}=3$ independent experiments. Values were given as means \pm SEM. $* P<0.05$ and $* * P<0.01$.

\section{Down-regulation of miR-455-3p abolishes DMY's inhibitory effect on cell proliferation and EMT in CCA cells}

In order to confirm that DMY inhibited EMT in CCA was through regulating miR-455-3p, we tested if inhibition of miR-455-3p expression using 455-i would abolish DMY's anti-tumor effects in CCA cell lines. Figure $4 \mathrm{~A}$ and $4 \mathrm{~B}$ showed that down-regulation of miR-455-3p remarkably abolished the inhibitory effect of DMY on cell growth and proliferation in REB cells as assessed by the colony formation assay and EdU assay. In addition, the transwell assay in both RBE cells and TFK-1 cells revealed that inhibition of
miR-455 abrogated DMY-mediated suppressing effects on cell migration and invasion (Figure 4C). Consistently, compared to RBE or TFK-1 cells transfected with NS-i and treatment with DMY, Western blot showed that down-regulation of miR-455-3p increased the expressions of vimentin and ZEB1 in those DMY-treated cells, while decreased the expression of E-cadherin (Figure 4D). Taken together, these data demonstrated that down-regulation of miR-455-3p abolished the inhibitory effects of DMY on cell proliferation and EMT in CCA cells, indicating that the mechanism of DMY's inhibitory effect was through regulating miR-455-3p. 
A

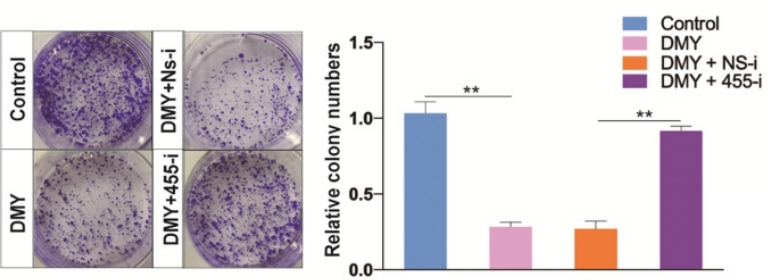

B
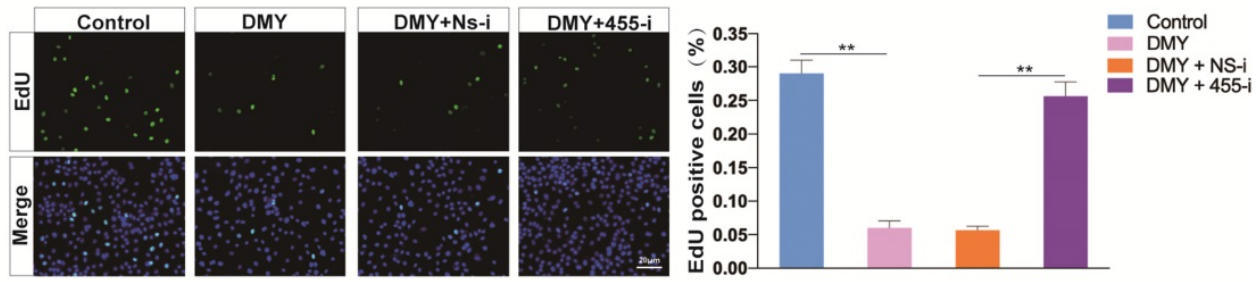

C
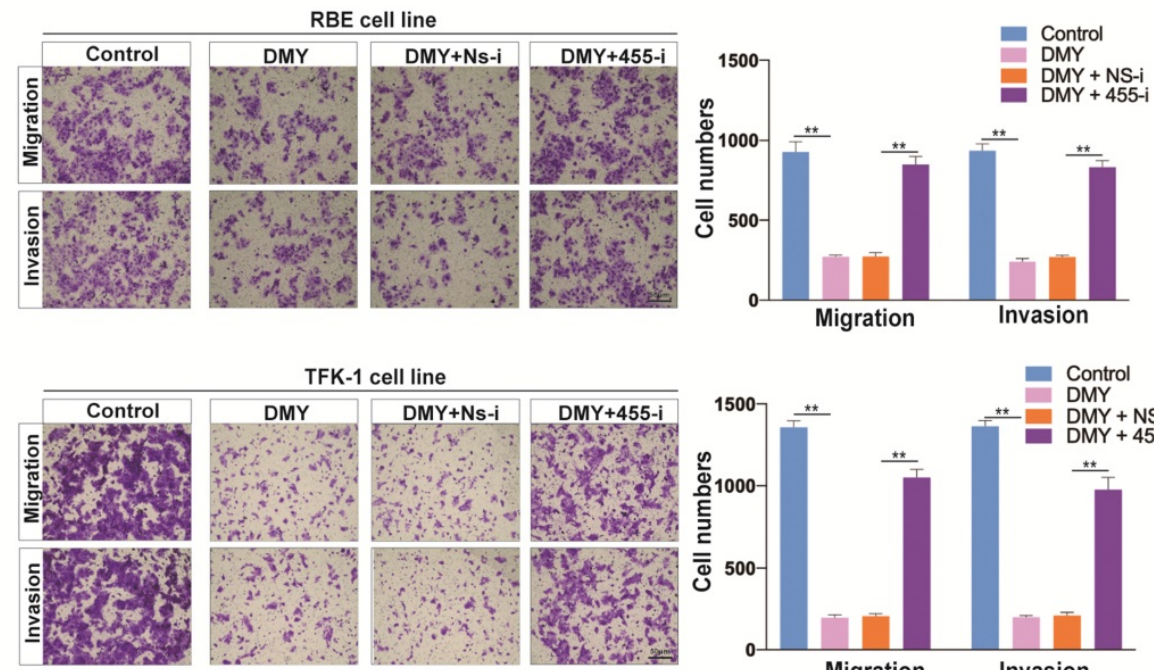

TFK-1 cell line
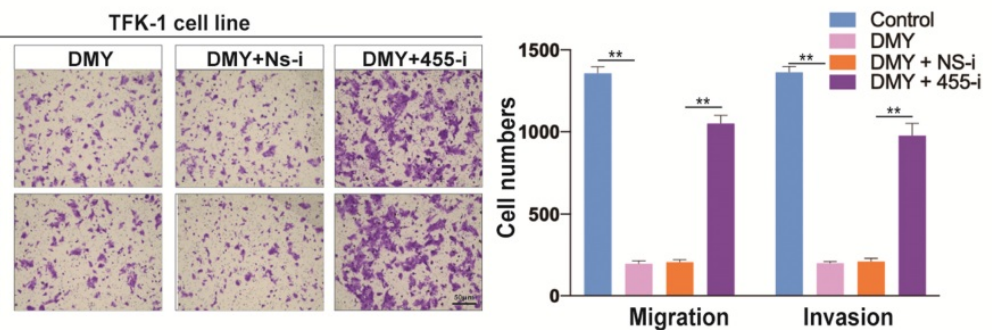

D
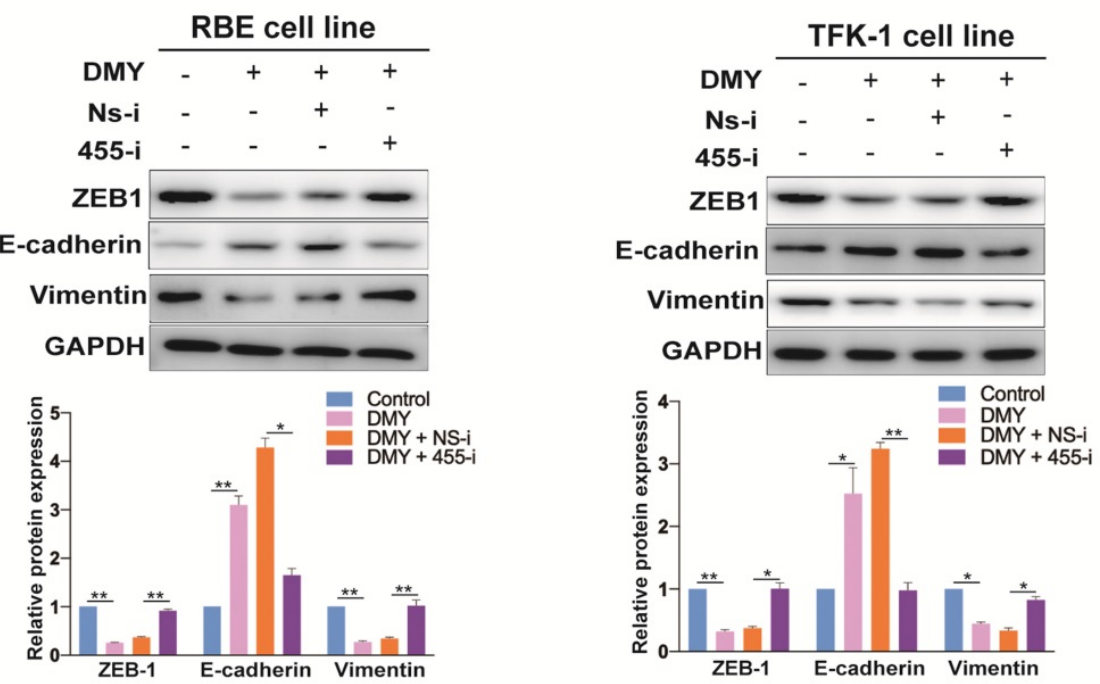

Figure 4. Downregulation of miR-455 abolishes dihydromyricetin' s inhibitory effect on cell proliferation and EMT in CCA cells. RBE cells and TFK-1 cells were transfected with miR-455-3p inhibitor (455-i) or non-specific inhibitor (NS-i). (A) Colony formation assay in RBE cells. (B) Edu assay in RBE cells. (C-D) Cell invasion and migration evaluated by transwell assay respectively in RBE cells and TFK-1 cells. (E) EMT related protein levels (ZEB1, E-cadherin, Vimentin) evaluated by Western blot assay in RBE cells and TFK-1 cells. $\mathrm{n}=3$ independent experiments. Values were given as means \pm SEM. $* P<0.05$ and $* * P<0.01$. 
A

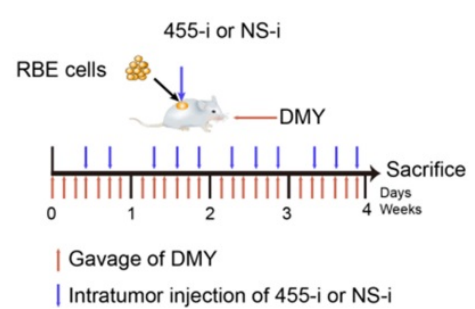

D
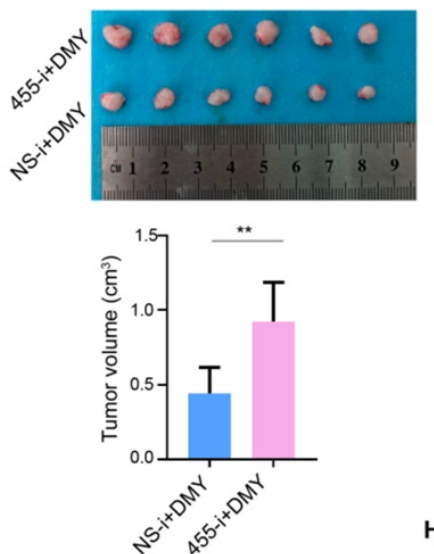

G

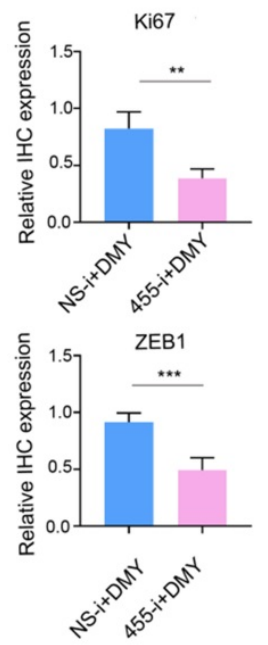

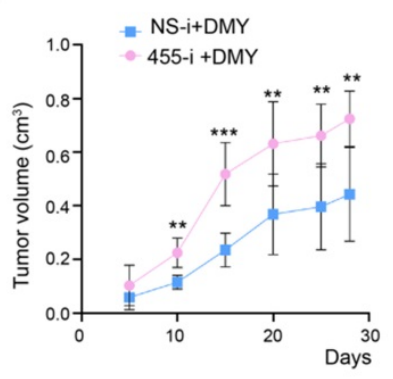

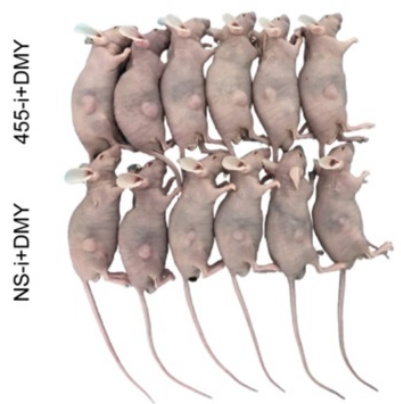

$\mathbf{F}$ ZEB1 staining
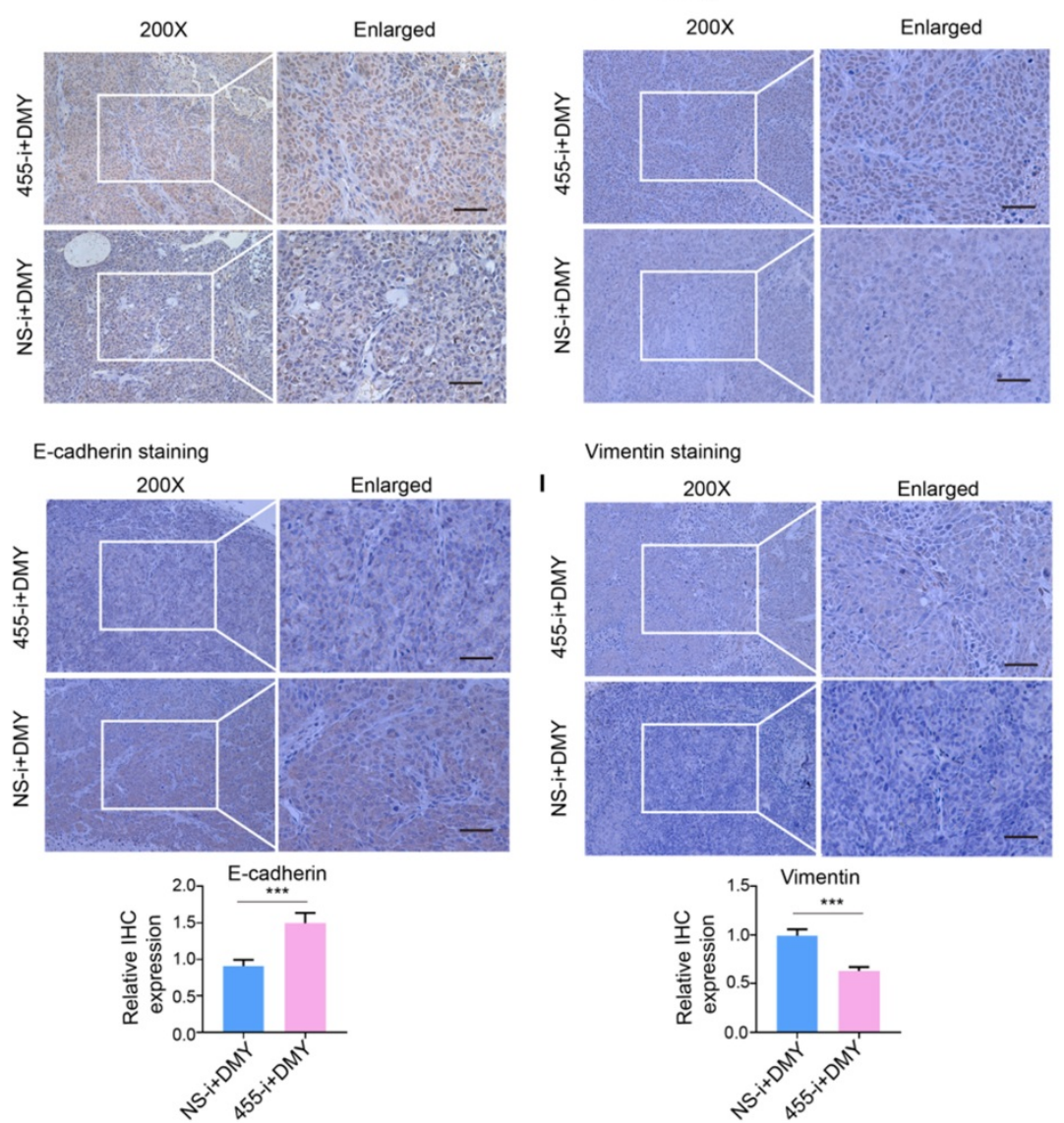
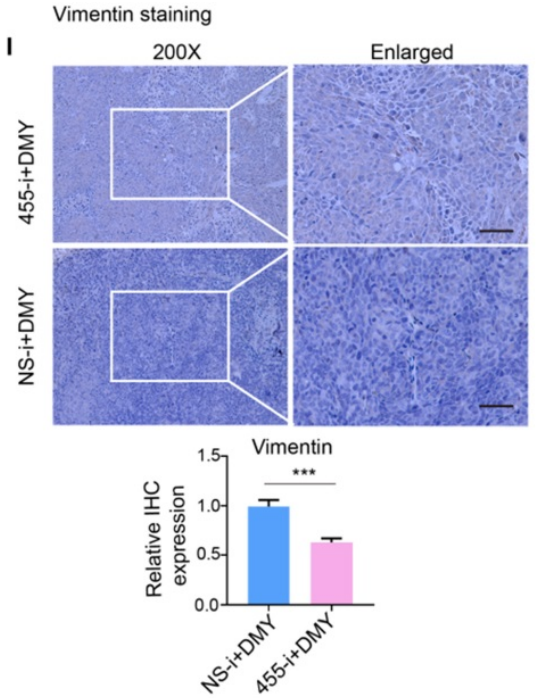

Figure 5. Inhibition of miR-455-3p abrogates dihydromyricetin-mediated inhibitory effects on tumor growth and EMT in human cholangiocarcinoma cell-engrafted nude mice. (A) Schematic graph illustrating the protocol of intratumoral injection with lipofectamine 2000 encapsulated miR-455-3p inhibitor (455-i) or non-specific inhibitor (NS-i) (5 $\mathrm{nM} /$ mouse) and gavaged with $500 \mathrm{mg} / \mathrm{kg} /$ day dihydromyricetin for four weeks. (B) Tumor growth curve over time of human cholangiocarcinoma cell-engrafted nude mice treated with dihydromyricetin and NS-i or 455-i, respectively. (C-D) Tumor image and quantification of tumor volume after mice treated with dihydromyricetin and NS-i or 455-i on day 29, respectively. (E, F, H, I) Immunohistochemistry staining and quantification of Ki67 and ZEB1, E-cadherin and Vimentin in xenograft tissues after mice treated with dihydromyricetin and NS-i or 455-i, respectively. (G) Histograms of the relative IHC expression of Ki67 and ZEB1. Scar bar $100 \mu M$, insert $50 \mu M$. $n=6$ mice per group. Values are given as means \pm SEM. $* * P<0.01$ and $* * * P<0.001$.

\section{Inhibition of miR-455-3p abrogates DMY- mediated inhibitory effects on tumor growth and EMT in human CCA cell-engrafted nude mice}

Subsequently, we further investigated the role of miR-455-3p in DMY-mediated EMT inhibitory effect in human CCA cell-engrafted nude mice. RBE cells (5 $\times 10^{6}$ ) were subcutaneously injected into four-week old Balb/c male nude mice. Three days after injection, the mice were randomly divided into two groups and gavaged with $500 \mathrm{mg} / \mathrm{kg} /$ day DMY for four weeks. Figure 5A describes the protocol of intratumoral injection with lipofectamine 2000 encapsulated 455-i or NS-i ( $5 \mathrm{nM} /$ mouse). On day 29 , the mice were sacrificed and tumors were harvested for further analyses. As shown in Figure 5B, the tumors grew much faster in mice injected with 455-i compared to the paired mice injected with NS-i. Figure 5C and 5D showed that the tumor volumes in the group injected with 455-i were significantly larger those injected with NS-i. Moreover, the immunohistochemistry staining 
and quantification of xenograft tissues showed that down-regulation of miR-455-3p in vivo significantly increased the protein expressions of ki67, vimentin and ZEB1, while decreased the protein level of E-cadherin (Figure 5E-H). Collectively, these data demonstrated that inhibition of miR-455-3p abrogated DMY-mediated inhibitory effects on tumor growth and EMT in human CCA cell-engrafted nude mice, further supporting the mechanism of DMY's inhibitory effect on tumor growth and EMT in human CCA was through regulating miR-455-3p.

\section{DMY inhibits the PI3K/AKT signaling pathway through regulating miR-455-3p in CCA cells}

Previous studies have demonstrated that the ZEB1/PI3K/AKT signaling pathway plays an important role in cell growth and invasion in several cancers $[19,20]$. Thus, we were interested to investigate if DMY could inhibit the ZEB1 downstream PI3K/AKT signaling pathway through regulating miR-455-3p in CCA. As shown in Figure 6, compared to the control cells, DMY treatment significantly suppressed the expression ratio of the phosphorylated PI3K (p-PI3K) to total PI3K (t-PI3K), and the expression ratio of the phosphorylated AKT (p-AKT) to total AKT (t-AKT) was also remarkably attenuated following DMY treatment in both RBE cells and TFK-1 cells as determined by Western blot. However, compared to cells transfected with NS-i, cells transfected with 455-i remarkably enhanced the expression ratios of both $\mathrm{p}-\mathrm{PI} 3 \mathrm{~K}$ to t-PI3K and $\mathrm{p}-\mathrm{AKT}$ to $\mathrm{t}-\mathrm{AKT}$, indicating that down-regulation of miR-455-3p reversed the inhibitory effect of DMY on

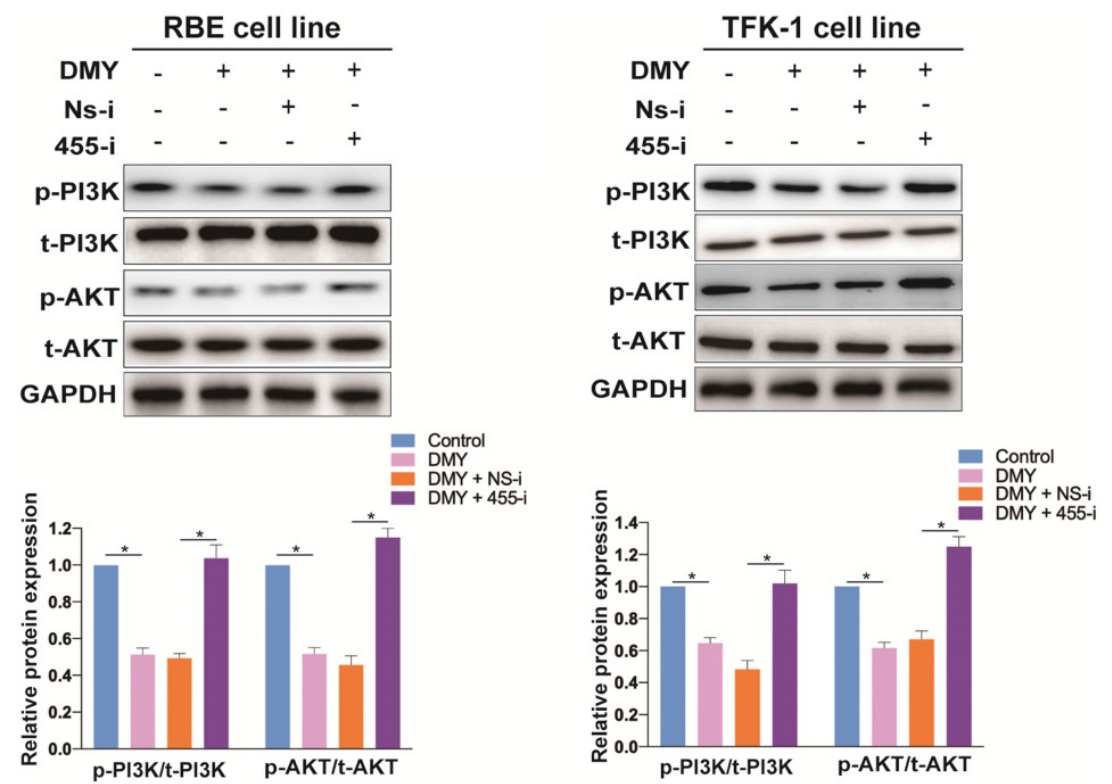

Figure 6. Dihydromyricetin inhibits the PI3K/AKT signaling pathway through regulating miR-455-3p in CCA cells. RBE cells and TFK-1 cells were transfected with miR-455-3p inhibitor (455-i) or non-specific inhibitor (NS-i). Protein expressions were evaluated by Western blot. $n=3$ independent experiments. Values were given as means \pm SEM. $* P<0.05$. the PI3K/AKT signaling pathway. In brief, our study confirmed that DMY inhibited EMT in CCA through regulation of the miR-455/ PI3K/AKT pathway.

\section{Discussion}

With advance in the understanding of molecular mechanisms of tumorigenesis and tumor progression, the discovery and development of bioactive compounds from natural plants for the treatment of different cancers has aroused great interest of scientists and healthcare workers [21, 22]. DMY, the most abundant natural flavonoid and active compound in Ampelopsis grossedentata W.T. Wang (Vitaceae), has been used as herbal tea and traditional Chinese medicines for over hundreds of years in China. In the past decades, DMY has been widely investigated and shows broad anti-tumor effects. For instance, DMY enhanced the anti-tumor effect of irinotecan in colon cancer in mouse models [8]. Wang et al showed that DMY reduced cell migration and invasion and induced cell apoptosis through downregulation of the golgi reassembly and stacking protein 65 (GRASP65) in human ovarian cancer cells [9]. In our previous study, we identified that DMY significantly inhibited cell proliferation, migration, invasion and promoted apoptosis in human CCA cell lines via regulating the miR-21/ PTEN/ Akt pathway [11]. In this study, we not only confirmed the anti-tumor effect of DMY in a human CCA cellengrafted nude mice model, but also showed that DMY inhibited EMT through targeting miR-455/ ZEB1 axis, an important mechanism associated with tumor initiation and progression in various cancer types, in human CCA both in vitro and in vivo experiments. This study provides novel evidence that the anti-cancer properties of DMY in CCA through inhibiting EMT, indicating that DMY may be an alternative option for the future treatment of CCA.

The characteristics of high aggressiveness and chemoresistance of CCA contribute to the poor prognosis. Therefore, it is very important to develop new treatment agents based on the understanding of pathogenic molecular mechanisms of CCA. EMT is a biological program involved in the process of organ development and wound healing. However, when EMT is hijacked by cancer cells, this process is often associated with increased tissue invasiveness, cancer stem cell 
characteristics and resistance to therapeutic regimens [23]. Studies have shown that the induction of EMT contributed to the progression of CCA [24], and CCA cells with EMT-like features could induce immunosuppression [25]. A review has extensively overviewed different EMT markers investigated in CCA [26]. When EMT occurs in cancer, epithelial cancer cells are detached from each other and the basement membrane, then epithelial cancer cells gradually obtain mesenchymal traits with invasive properties after a new transcriptional program is activated, resulting in greater potential of metastatic colonization and therapy resistance [27]. Initially believed as a binary process, the recent researches have demonstrated that EMT occurs in a dynamic process which confers to intermediate cellular states with both epithelial and mesenchymal features, leading to cell heterogeneity, tumorigenesis and cancer progression $[27,28]$.

EMT is regulated by transcription factors (EMT-TFs), including zinc finger proteins (e.g., SNAI1 and SNAI2), basic helix-loop-helix transcription factors (e.g., the TWIST family and E47) and zinc finger and homeodomain proteins (ZEB1 and ZEB2) [29]. In this study, we performed the relationship analyses between the expressions of the EMT-TF and miR-455 in human CCA tissues by using the TCGA dataset. As shown in Figure S3, the expression of neither SNAIL nor TWIST was statistically related to the level of miR-455, while the expression of ZEB1 was negatively associated with the expression of miR-455. Therefore, we focused on ZEB1. The Kaplan-Meier analysis (Figure S3) did not demonstrate positive correlation between miR-455-3P (or ZEB1) and CCA patient survival time, probably these was due to small sample sizes. Larger clinical samples are required to verify the results. ZEB1 is considered as a driver of EMT and cancer progression. For example, ZEB1 could down-regulate the expression of E-cadherin, a major cell-cell adhesion molecule, and up-regulate the expression of vimentin, a key protein for EMT and cancer metastasis [29, 30]. In our study, the functional experiments of the transwell assays demonstrated that DMY treatment suppressed the migration and invasion of CCA cell lines, which in line with EMT inhibition, indicated by decreased ZEB1 and its downstream protein of vimentin expression and upregulated E-cadherin expression in CCA cells treatment with DMY.

MiRNAs act as oncogenes or tumor suppressor genes in controlling tumor initiation, progression and metastasis. The role of miR-455 has been investigated in several cancers. For instance, in human breast cancer cells, miR-455 inhibited cell proliferation through targeting CDK14 [31]. MiR-455 suppressed cell growth and invasion through targeting SMAD2 in pancreatic cancer cells. Moreover, this study found that low expression of miR-455 and high level of SMAD2 were associated with poor patient survival [22]. In non-small cell lung cancer, miR-455 also exhibited anti-tumor property. MiR-455 inhibited the proliferation, migration, and invasion of non-small cell lung cancer cell lines by targeting ZEB1. Overexpression of ZEB1 reversed the inhibitory effect of miR-455 [16]. Similarly, a recent study also showed that miR-455 could inhibit EMT through targeting ZEB1 in arsenite-induced malignant transformation of human keratinocytes [18]. However, the role of miR-455 in CCA is still not clear. Our study found that miR-455 was down-regulated in CCA tissues compared to normal tissues, and overexpression of miR-455-3p inhibited ZEB1 expression in human CCA. Importantly, our study demonstrated that DMY increased the expression of miR-455-3p, while decreased the expression of ZEB1. In support, down-regulation of miR-455-3p expression abolished the inhibitory effects of DMY on cell proliferation and EMT in vitro and in vivo. Together with our previous study [11], our data indicated that the anti-tumor effects of DMY's on CCA is through a synthetic mechanism associated with multiple targets, such as miR-455 and miR-21.

The PI3K/AKT signaling pathway contributes importantly to tumor proliferation and metastasis in multiple human malignancies, including CCA [32, 33]. Anti-tumor agents such as anlotinib inhibits tumor progression via targeting the PI3K/AKT pathway in intrahepatic cholangiocarcinoma [34]. AGO1 enhances proliferation and invasion through modulating EMT-associated related TGF- $\beta$-PI3K-AKT signaling pathways in cholangiocarcinoma [5]. The ZEB1/PI3K/AKT signaling pathway also plays an important role in tumor cell growth and invasion [19, 20]. For instance, in non-small cell lung cancer cells, downregulation of Rhomboid domain containing 1 (RHBDD1) expressions inhibits cell growth and invasion through reducing the ZEB1/PI3K/AKT signaling pathway activation [19]. In the current study, we showed that DMY treatment inhibited the expression of ZEB1, p-PI3K and p-AKT in both RBE cells and TFK-1 cells, which in line with increased miR-455 expression. In agreement with this observation, inhibition of miR-455 abrogated the anti-tumor effects of DMY on CCA, as well as ZEB1, p-PI3K and p-AKT expression, indicating that DMY inhibits EMT in CCA through targeting the miR-455/ PI3K/AKT pathway (Figure S4).

\section{Conclusion}

In conclusion, our study demonstrated that DMY 
suppressed cell proliferation, tumor growth and EMT through regulating miR-455-3p in CCA. We believe this study will help us to better understand the effects of DMY in human CCA and these findings suggest that DMY may be explored as a therapeutic option for the treatment of CCA.

\section{Abbreviations}

CCA: cholangiocarcinoma; CCK-8: Cell Counting Kit-8; DMY: Dihydromyricetin; ECL: electrochemiluminescence; EMT: epithelialmesenchymal transition; IC50: half maximal inhibitory concentration; MET: mesenchymalepithelial transition; NS-i: non-specific inhibitors; NS-m: non-specific mimics; 455-m: miR-455-3p mimics; 455-i: miR-455-3p inhibitors; PVDF: polyvinylidene fluoride; SDS-PAGE: sodium dodecyl sulfate - polyacrylamide gel electrophoresis; TF: transcription factor.

\section{Supplementary Material}

Supplementary figures.

http://www.jcancer.org/v12p6058s1.pdf

\section{Acknowledgements}

This work was supported by the National Natural Science Foundation of China (grant number 81703818, 81400331 and 81703767), the Natural Science Foundation of Hunan Province (grant number 2018JJ3731, 2019JJ40430, 2019JJ50680 and 2019JJ50891), $\mathrm{Wu}$ Jieping Medical Foundation (grant number 320.6750.2020-4-35 and 320.6750.2020-4-36) and the Self-financing Scientific Research Project of Guangxi Health Commission (grant number Z20201003).

\section{Competing Interests}

The authors have declared that no competing interest exists.

\section{References}

1. Rizvi S, Khan SA, Hallemeier CL, Kelley RK, Gores GJ. Cholangiocarcinoma - evolving concepts and therapeutic strategies. Nat Rev Clin Oncol. 2018;15(2):95-111.

2. Banales JM, Marin JJG, Lamarca A, Rodrigues PM, Khan SA, Roberts LR, et al. Cholangiocarcinoma 2020: the next horizon in mechanisms and management. Nat Rev Gastroenterol Hepatol. 2020;17(9):557-88.

3. Forner A, Vidili G, Rengo M, Bujanda L, Ponz-Sarvise M, Lamarca A. Clinical presentation, diagnosis and staging of cholangiocarcinoma. Liver Int. 2019;39 Suppl 1:98-107.

4. Lamouille S, Xu J, Derynck R. Molecular mechanisms of epithelialmesenchymal transition. Nat Rev Mol Cell Biol. 2014;15(3):178-96.

5. Wu G, Fan F, Hu P, Wang C. AGO1 enhances the proliferation and invasion of cholangiocarcinoma via the EMT-associated TGF-beta signaling pathway. Am J Transl Res. 2020;12(6):2890-902.

6. Zou Z, Zheng B, Li J, Lv X, Zhang H, Yu F, et al. TPX2 level correlates with cholangiocarcinoma cell proliferation, apoptosis, and EMT. Biomed Pharmacother. 2018;107:1286-93.

7. Avila-Carrasco L, Majano P, Sanchez-Tomero JA, Selgas R, Lopez-Cabrera M, Aguilera A, et al. Natural Plants Compounds as Modulators of Epithelial-to-Mesenchymal Transition. Front Pharmacol. 2019;10:715.
8. Zhu XH, Lang HD, Wang XL, Hui SC, Zhou M, Kang C, et al. Synergy between dihydromyricetin intervention and irinotecan chemotherapy delays the progression of colon cancer in mouse models. Food Funct. 2019;10(4):2040-9.

9. Wang F, Chen X, Yuan D, Yi Y, Luo Y. Golgi reassembly and stacking protein 65 downregulation is required for the anti-cancer effect of dihydromyricetin on human ovarian cancer cells. PLoS One. 2019;14(11):e0225450.

10. Han JM, Kim HL, Jung HJ. Ampelopsin Inhibits Cell Proliferation and Induces Apoptosis in HL60 and K562 Leukemia Cells by Downregulating AKT and NF-kappaB Signaling Pathways. Int J Mol Sci. 2021;22(8):4265.

11. Chen L, Yang ZS, Zhou YZ, Deng Y, Jiang P, Tan SL. Dihydromyricetin inhibits cell proliferation, migration, invasion and promotes apoptosis via regulating miR-21 in Human Cholangiocarcinoma Cells. J Cancer. 2020;11(19):5689-99.

12. Zhang S, Xiao J, Chai Y, Du YY, Liu Z, Huang K, et al. LncRNA-CCAT1 Promotes Migration, Invasion, and EMT in Intrahepatic Cholangiocarcinoma Through Suppressing miR-152. Dig Dis Sci. 2017;62(11):3050-8.

13. Zhang M, Shi B, Zhang K. miR-186 Suppresses the Progression of Cholangiocarcinoma Cells Through Inhibition of Twist1. Oncol Res. 2019;27(9):1061-8

14. Zhang S, Yin WL, Zhang X, Zhang XY. MicroRNA455 is downregulated in gastric cancer and inhibits cell proliferation, migration and invasion via targeting insulinlike growth factor 1 receptor. Mol Med Rep. 2017;16(3):3664-72.

15. Xu B, Gong X, Zi L, Li G, Dong S, Chen X, et al. Silencing of DLEU2 suppresses pancreatic cancer cell proliferation and invasion by upregulating microRNA-455. Cancer Sci. 2019;110(5):1676-85.

16. Li YJ, Ping C, Tang J, Zhang W. MicroRNA-455 suppresses non-small cell lung cancer through targeting ZEB1. Cell Biol Int. 2016;40(6):621-8.

17. Sun X, He S, Wara AKM, Icli B, Shvartz E, Tesmenitsky Y, et al. Systemic delivery of microRNA-181b inhibits nuclear factor-kappaB activation, vascular inflammation, and atherosclerosis in apolipoprotein E-deficient mice. Circ Res. 2014;114(1):32-40.

18. Xue J, Chen C, Luo F, Pan X, Xu H, Yang P, et al. CircLRP6 Regulation of ZEB1 via miR-455 Is Involved in the Epithelial-Mesenchymal Transition During Arsenite-Induced Malignant Transformation of Human Keratinocytes. Toxicol Sci. 2018;162(2):450-61.

19. Xu Z, Wang R, Li X, Yang L, Peng H, Wang Y, et al. RHBDD1 silencing inhibited cell growth and invasion of non-small cell lung cancer by mediating ZEB1/PI3K/AKT signaling pathway. J Mol Histol. 2021;52(3):503-10.

20. Liao LZ, Chen CT, Li NC, Lin LC, Huang BS, Chang YH, et al. Y-Box Binding Protein-1 Promotes Epithelial-Mesenchymal Transition in Sorafenib-Resistant Hepatocellular Carcinoma Cells. Int J Mol Sci. 2020;22(1):224.

21. Mann J. Natural products in cancer chemotherapy: past, present and future. Nat Rev Cancer. 2002;2(2):143-8.

22. Mignani S, Rodrigues J, Tomas H, Zablocka M, Shi X, Caminade AM, et al. Dendrimers in combination with natural products and analogues as anti-cancer agents. Chem Soc Rev. 2018;47(2):514-32.

23. De Craene B, Berx G. Regulatory networks defining EMT during cancer initiation and progression. Nat Rev Cancer. 2013;13(2):97-110.

24. Claperon A, Mergey M, Nguyen Ho-Bouldoires TH, Vignjevic D, Wendum D, Chretien $\mathrm{Y}$, et al. EGF/EGFR axis contributes to the progression of cholangiocarcinoma through the induction of an epithelial-mesenchymal transition. J Hepatol. 2014;61(2):325-32.

25. Qian Y, Yao W, Yang T, Yang Y, Liu Y, Shen Q, et al. aPKC-iota/P-Sp1/Snail signaling induces epithelial-mesenchymal transition and immunosuppression in cholangiocarcinoma. Hepatology. 2017;66(4):1165-82.

26. Vaquero J, Guedj N, Claperon A, Nguyen Ho-Bouldoires TH, Paradis V, Fouassier L. Epithelial-mesenchymal transition in cholangiocarcinoma: From clinical evidence to regulatory networks. J Hepatol. 2017;66(2):424-41.

27. Dongre A, Weinberg RA. New insights into the mechanisms of epithelial-mesenchymal transition and implications for cancer. Nat Rev Mol Cell Biol. 2019;20(2):69-84.

28. Pastushenko I, Blanpain C. EMT Transition States during Tumor Progression and Metastasis. Trends Cell Biol. 2019;29(3):212-26.

29. Caramel J, Ligier M, Puisieux A. Pleiotropic Roles for ZEB1 in Cancer. Cancer Res. 2018;78(1):30-5.

30. Zhang P, Sun Y, Ma L. ZEB1: at the crossroads of epithelial-mesenchymal transition, metastasis and therapy resistance. Cell Cycle. 2015;14(4):481-7. 
31. Wang B, Zou A, Ma L, Chen X, Wang L, Zeng X, et al. miR-455 inhibits breast cancer cell proliferation through targeting CDK14. Eur J Pharmacol. 2017;807:138-43.

32. Tiemin $P$, Fanzheng $M$, Peng $X$, Jihua $H$, Ruipeng S, Yaliang L, et al. MUC13 promotes intrahepatic cholangiocarcinoma progression via EGFR/PI3K/AKT pathways. J Hepatol. 2020;72(4):761-73.

33. Zhang Y, Ji G, Han S, Shao Z, Lu Z, Huo L, et al. Tip60 Suppresses Cholangiocarcinoma Proliferation and Metastasis via PI3k-AKT. Cell Physiol Biochem. 2018;50(2):612-28.

34. Song F, Hu B, Cheng JW, Sun YF, Zhou KQ, Wang PX, et al. Anlotinib suppresses tumor progression via blocking the VEGFR2/PI3K/AKT cascade in intrahepatic cholangiocarcinoma. Cell Death Dis. 2020;11(7):573. 